\title{
Antinociceptive, anti-inflammatory and antipyretic properties of Melastoma malabathricum leaves aqueous extract in experimental animals
}

\begin{abstract}
The present study was carried out to establish the antinociceptive, anti-inflammatory, and antipyretic properties of the aqueous extract of Melastoma malabathricum leaves in experimental animals. The antinociceptive activity was measured using abdominal constriction, hot-plate, and formalin tests, whereas the anti-inflammatory and antipyretic activities were measured using carrageenan-induced paw edema and brewerố yeast-induced pyrexia tests, respectively. The extract, which was obtained after soaking the air-dried leaves in distilled water for $72 \mathrm{~h}$ and then preparing in concentrations of $10 \%, 50 \%$, and $100 \%(\mathrm{v} / \mathrm{v})$, was administered subcutaneously 30 min prior to subjection to the above mentioned assays. At all concentrations tested, the extract was found to exhibit significant $(\mathrm{P}<0.05)$ antinociceptive, anti-inflammatory, and antipyretic activities in a concentration-independent manner. Our findings that the aqueous extract of $M$. malabathricum possesses antinociceptive, anti-inflammatory, and antipyretic activities supports previous claims on its traditional uses to treat various ailments.
\end{abstract}

Keyword: Anti-inflammatory; Antinociceptive; Antipyretic; Aqueous extract; Melastoma malabathricum 